\title{
Fluorescence-based microtiter plate assay for glutamate-cysteine ligase activity
}

\author{
Collin C. White, Hannah Viernes, Cecile M. Krejsa, ${ }^{1}$ Dianne Botta, \\ and Terrance J. Kavanagh*
}

NIEHS Center Ecogenetics and Environmental Health, Department of Environmental Health, University of Washington, Seattle, WA 98195, USA

Received 24 May 2002

\begin{abstract}
Glutamate-cysteine ligase (GCL; also known as $\gamma$-glutamylcysteine synthetase) is the rate-limiting enzyme in glutathione (GSH) synthesis. Traditional assays for the activity of this enzyme are based either on coupled reactions with other enzymes or on highperformance liquid chromatography (HPLC) assessment of $\gamma$-glutamylcysteine $(\gamma$-GC) product formation. We took advantage of the reaction of naphthalene dicarboxaldehyde (NDA) with GSH or $\gamma$-GC to form cyclized products that are highly fluorescent. Hepa-1 cells which were designed to overexpress mouse GCL and mouse liver homogenates were used to evaluate and compare the utility of the NDA method with an assay based on monobromobimane derivatization and HPLC analysis with fluorescence detection. Excellent agreement was found between GCL activities measured by HPLC and NDA-microtiter plate analyses. This assay should be useful for high-throughput GCL activity analyses.
\end{abstract}

(c) 2003 Elsevier Science (USA). All rights reserved.

Keywords: Glutamate-cysteine ligase; Glutathione; Microtiter plate; Fluorescence

The antioxidant and free radical scavenger glutathione is important in the preservation of cellular redox status and defense against reactive oxygen species and xenobiotics [1]. Glutathione is a tripeptide composed of glutamic acid, cysteine, and glycine. Under normal conditions, and given adequate cysteine levels, the ratelimiting enzyme in GSH synthesis is glutamate-cysteine ligase (GCL; ${ }^{2}$ EC 6.3.2.2; also known as $\gamma$-glutamylcysteine synthetase).

GCL is a heterodimer consisting of a $72-\mathrm{kDa}$ catalytic subunit (GCLC) and a $30-\mathrm{kDa}$ subunit (GCLM).

\footnotetext{
${ }^{*}$ Corresponding author. Fax: 1-206-685-4696.

E-mail address: tjkav@u.washington.edu (T.J. Kavanagh).

${ }^{1}$ Current address: Cerep, Inc., Redmond, WA, USA.

2 Abbreviations used: ANOVA, analysis of variance; DMEM, Dulbecco's modified Eagle's medium; $\gamma$-GC, $\gamma$-glutamylcysteine; GCL, glutamate-cysteine ligase; GCLC, glutamate-cysteine ligase catalytic subunit; GCLM, glutamate-cysteine ligase modulatory subunit; GS, glutathione synthetase; MCB, monochlorobimane; MBB, monobromobimane; NDA, 2,3-naphthalenedicarboxaldehyde; SSA, 5-sulfosalicylic acid; PBS, phosphate-buffered saline; SB, serine borate; TES, Tris, ETDA, sucrose; BSO, buthionine- $S, R$-sulfoximine; GGT, $\gamma$-glutamyltranspeptidase.
}

GCLM modifies the catalytic activity of the holoenzyme such that the $K_{\mathrm{m}}$ for glutamate is lowered and the $K_{\mathrm{i}}$ for GSH is increased [2,3]. Rat, human, and mouse cDNAs for both subunits have been cloned [2,4-9], and both subunit genes have been mapped on human and mouse chromosomes $[10,11]$. GCL expression can be modulated by a number of different factors, including depleting agents, reactive oxygen and nitrogen species, cytokines, and hormones [reviewed in 12].

Many different assays of GCL activity have been described [13-19], which in general rely on spectrophotometric detection of a secondary analyte or on HPLC analysis of thiols. The most popular spectrophotometric assay for GCL activity is based upon the coupling of GCL enzyme activity to an ATP-generating system which, in turn, consumes NADPH reducing equivalents; NADPH is then easily monitored by absorption at $340 \mathrm{~nm}$ [20]. HPLC-based assays are usually conducted with UV, fluorometric, or electrochemical detection of the $\gamma$-glutamylcysteine $(\gamma$-GC) peak in the chromatogram. While spectrophotometric assays can be conveniently adapted to $96-w e l l$ plate readers, this tends to 
decrease the sensitivity of the assay for tissues/cell types having low GCL activity.

A fluorescence 96-well plate assay for GCL activity has been described by Nelson and colleagues [21]. This assay is based on the detection of glutathione $S$-transferase-mediated conjugation of monochlorobimane (MCB) to GSH and so provides an indirect measure of GCL activity. In addition, because it relies on conversion of $\gamma$-GC to GSH by glutathione synthetase (GS) present in the preparation, under certain circumstances, GS may be limiting and therefore the assay would not be reporting GCL activity, but rather GS activity.

Most fluorometric HPLC assays of GCL activity depend upon the derivatization of thiols with either bimane or $o$-phthaldialdehyde, followed by separation of the thiol derivatives and specific quantitation of derivatized $\gamma$-GC. While highly specific and sensitive, HPLC assays of GCL activity suffer from lack of convenience and low throughput.

Recently, Orwar and colleagues [22] reported that 2,3-naphthalenedicarboxaldehyde (NDA) could be used to measure GSH with high specificity, because of its ability to form a cyclic reaction product with the cysteine sulfhydryl group and the glutamyl amino group of GSH. A cyclic compound with essentially equivalent fluorescence characteristics can also result from the reaction of NDA with $\gamma$-GC. The excitation and emission maxima of these reaction products are conveniently centered around those of fluorescein and thus provide for analyses in instruments that use argon-ion laser light sources or in fluorescence microtiter plate readers which use standard fluorescein excitation/emission optics. We describe here an assay of GCL activity that combines the sensitivity of fluorometric detection and the convenience of a 96-well (or 384-well) microtiter plate format. This assay shows high specificity for GCL activity and has the advantage that baseline levels of GSH can also be measured in the same cell/tissue samples.

\section{Materials and methods}

\section{Chemicals and reagents}

2,3-Naphthalenedicarboxyaldehyde was purchased from Aldrich Chemical (Sigma-Aldrich, St. Louis, MO, USA). Monobromobimane (Thiolyte) was from Calbiochem (La Jolla, CA, USA). Glutathione, $\gamma$-glutamylcysteine, ATP, 5-sulfosalicylic acid (SSA), L-glutamic acid, L-cysteine, and other common reagents were purchased from Sigma Chemical Co. (St. Louis, MO, USA).

\section{Sample preparation}

Cells and culture. The mouse Gclc and Gclm cDNAs $[7,8]$ were cloned into plasmid constructs containing a metallothionein promoter [23] and transfected into mouse Hepa-1c1c7 hepatoma cells (Hepa-1). A clonal Hepa-1 cell line overexpressing both GCLC and GCLM (CR17 cells) and a cell line containing the plasmid vector alone (Hepa-V cells) were used in these studies. Untreated CR17 cells and $\mathrm{ZnCl}_{2}$-treated CR17 cells contain approximately 2.5 and 5 times the GCL activity of untreated Hepa-V cells, respectively (see below). Cells were grown in DMEM/F12 medium with $10 \% \mathrm{Nu}$ serum (Collaborative Biomedical Products, Bedford, MA, USA) at $37^{\circ} \mathrm{C}$ in a humidified atmosphere containing $5 \% \mathrm{CO}_{2} / 95 \%$ air. For assay of GCL activity, cells were trypsinized and then triturated in medium with $\mathrm{Nu}$ serum. The cell suspensions were then placed in $15-\mathrm{ml}$ polystyrene tubes and pelleted in a Beckman tabletop centrifuge. Cell pellets were then resuspended in $500 \mu 1$ PBS, transferred to microfuge tubes, and sonicated to disrupt cell membranes (three 5-s bursts, on ice). Sonicates were then centrifuged to remove cellular debris, and the supernatants were transferred to wells of microtiter plates.

Mouse liver samples. Mouse liver samples were obtained from C57Bl/6 mice fed ad libitum on standard lab chow. In some cases, mice were injected with diethylmaleate to deplete liver GSH. Animals were sacrificed by $\mathrm{CO}_{2}$ narcosis followed by cervical dislocation, in accordance with University of Washington Institutional Animal Care and Use Committee procedures. Pieces of liver were homogenized in TES/SB buffer $(20 \mathrm{mM}$ Tris, $1 \mathrm{mM}$ EDTA, $250 \mathrm{mM}$ sucrose, $20 \mathrm{mM}$ sodium borate, $2 \mathrm{mM}$ serine) $1 / 4 \mathrm{w} / \mathrm{v}$. Homogenates were then centrifuged at $4^{\circ} \mathrm{C}$ for $10 \mathrm{~min}$ at $10,000 \mathrm{~g}$. Supernatants were then transferred to additional tubes and centrifuged at $4{ }^{\circ} \mathrm{C}$ for $20 \mathrm{~min}$ at $15,000 \mathrm{~g}$. The sample supernatants were then diluted (see below) and transferred into a 96well microtiter plate.

Sample distribution. Each sample was aliquoted into duplicate wells $(250 \mu \mathrm{l} /$ well), each of which serves as a source for further distribution of the samples into the GCL reaction plate. Thus, pipetting from these duplicate wells four times $(50 \mu \mathrm{l} /$ transfer $)$ into a reaction plate yielded eight replicate samples, four of which were used for GCL activity measurements, and the remaining four wells served for baseline glutathione measurements. For ease of pipetting, a multichannel transfer pipette was used throughout the remaining steps of the assay.

\section{Enzyme activity and baseline glutathione determination}

On a separate 96-well round-bottom reaction plate, $50-\mu 1$ aliquots of GCL reaction cocktail $(400 \mathrm{mM}$ Tris, $40 \mathrm{mM}$ ATP, $20 \mathrm{mM}$ L-glutamic acid, $2.0 \mathrm{mM}$ EDTA, $20 \mathrm{mM}$ sodium borate, $2 \mathrm{mM}$ serine, $40 \mathrm{mM} \mathrm{MgCl}_{2}$ ) were pipetted into wells, 8 wells per sample. These plates were either prepared just prior to the assay or stored on ice to prevent degradation of ATP in the reaction 
cocktail. While the sample plate was kept on ice, $50-\mu 1$ aliquots of sample were pipetted into the prewarmed $\left(37^{\circ} \mathrm{C}\right)$ reaction plate at 15 -s time intervals. After a 5min preincubation, the GCL reaction was initiated by adding $50 \mu \mathrm{l}$ of $2 \mathrm{mM}$ cysteine (dissolved in TES/SB) to each GCL activity well (cysteine was not added to the GSH-baseline wells at this time). The plate was then vortexed, covered, and incubated for varying lengths of time (see below). The GCL reaction was stopped by adding $50 \mu 1200 \mathrm{mM}$ SSA to all wells, and then $50 \mu \mathrm{l}$ of $2 \mathrm{mM}$ cysteine was added to the GSH-baseline wells. The plate was then vortexed and held on ice for at least $20 \mathrm{~min}$. Following protein precipitation, the plate was centrifuged for $5 \mathrm{~min}$ at $2500 \mathrm{rpm}$ on a Beckman tabletop centrifuge.

\section{NDA derivatization}

Following centrifugation, 20- $\mu$ l aliquots of supernatant from each well of the reaction plate were transferred to a 96-well plate designed for fluorescence detection (Phenix Research Products, Hayward, CA, USA; Cat. No. M-77260039). Next, $180 \mu 1$ of NDA derivatization solution $(50 \mathrm{mM}$ Tris, $\mathrm{pH} 10,0.5 \mathrm{~N} \mathrm{NaOH}$, and $10 \mathrm{mM}$ NDA in $\mathrm{Me}_{2} \mathrm{SO}$, v/v/v 1.4/0.2/0.2) was added to all wells of this plate. The plate was covered to protect the wells from room light and allowed to incubate at room temperature for $30 \mathrm{~min}$.

\section{$M B B$ derivatization and HPLC analyses}

The performance of the NDA-spectrofluorometric GCL activity assay was compared with HPLC-based analysis of GCL activity, as routinely performed in our laboratory. Briefly, subsequent to protein precipitation and centrifugation (above), 200- $\mu$ l aliquots of supernatant from each well of the reaction plate were transferred to microcentrifuge tubes and subsequently derivatized with monobromobimane as previously reported. Derivatized samples were then transferred to narrow-bore, $500-\mu 1$ capacity brown glass HPLC reaction vials. Samples were then analyzed for GCL activity by reverse-phase HPLC as previously described [24,25].

\section{Fluorescence quantitation}

Following incubation, NDA- $\gamma-\mathrm{GC}$ or NDA-GSH fluorescence intensity was measured (472 ex/528 em) on a fluorescence plate reader (either a Gemini Spectrum, Molecular Devices, Menlo Park, CA, USA, or a FluoroCount microplate reader, Packard Instrument Co., Meriden, CT, USA). Alternatively, the plate was read on an ACAS Ultima scanning laser cytometer (Meridian Instruments, Okemos, MI, USA), using the 488-nm line of an argon-ion laser for excitation and 530/20 bandpass filter to collect fluorescence emission.

\section{Statistical analyses}

Data obtained from fluorescence measurements were imported into Microsoft Excel (Microsoft, Redmond, WA, USA) for analyses, using the Data Analyses tool pack. Linear regression analysis was used to compare results of HPLC and fluorescence plate reader analyses. Differences in GCL activity in Hepa-V and CR17 cells were analyzed with ANOVA and Student's $t$ test.

\section{Results}

In order to determine the responsiveness of the fluorescence plate reader to NDA- $\gamma-\mathrm{GC}$ and NDA-GSH, we constructed a standard curve for each of these conjugates in 96-well microtiter plates using purified $\gamma$-GC and GSH (Fig. 1). The curves were linear from 2.5 to $50 \mathrm{nM}$. Moreover, we found that the fluorescence intensities of NDA- $\gamma-\mathrm{GC}$ and NDA-GSH conjugates were essentially the same.

In the work published by Orwar and colleagues [21], the authors noted a strong $\mathrm{pH}$ dependence on the reaction rate of NDA with GSH. We therefore examined both the rate of reaction and the stability of the NDAGSH conjugate under different $\mathrm{pH}$ conditions. Fig. 2 shows that the reaction of NDA with GSH at a $\mathrm{pH}$ value of 12.5 produces a strong fluorescence reaction, which peaks after approximately $10 \mathrm{~min}$, and then shows a slight decline in fluorescence with continued incubation. Area-under-curve calculations showed a maximum fluorescence yield at $\mathrm{pH}$ values above 11.5. Thus, we chose to derivatize GCL incubation reactions at $\mathrm{pH} 12.5$.

Fig. 3 shows a comparison of NDA-fluorescencebased and MBB-HPLC-based assays of GCL activity using cultured mouse liver cell lines expressing different

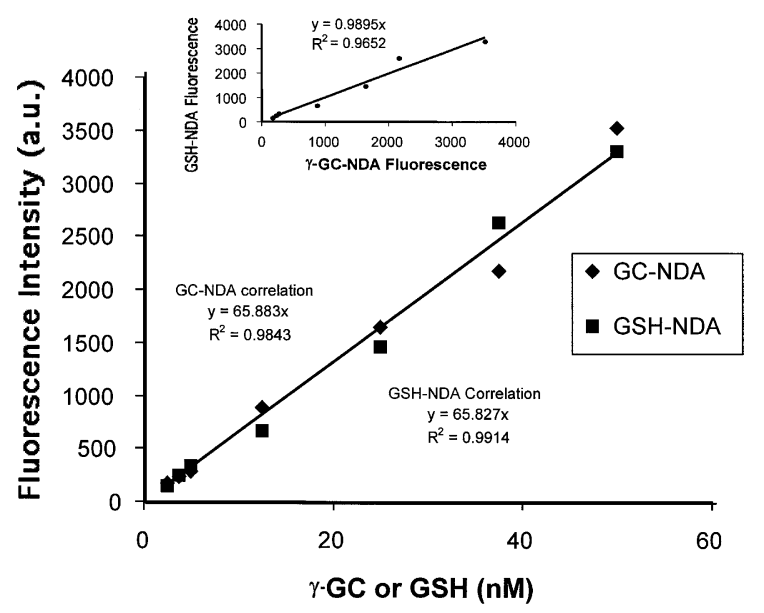

Fig. 1. Standard curves for NDA-GSH and NDA- $\gamma-$ GC fluorescence. Reagent-grade GSH and $\gamma$-GC were mixed with NDA as described under Materials and methods at $\mathrm{pH} 12.5$ and fluorescence was measured on a fluorescence plate reader. Inset: Correlation between NDAGSH and NDA- $\gamma$-GC standard curves. 


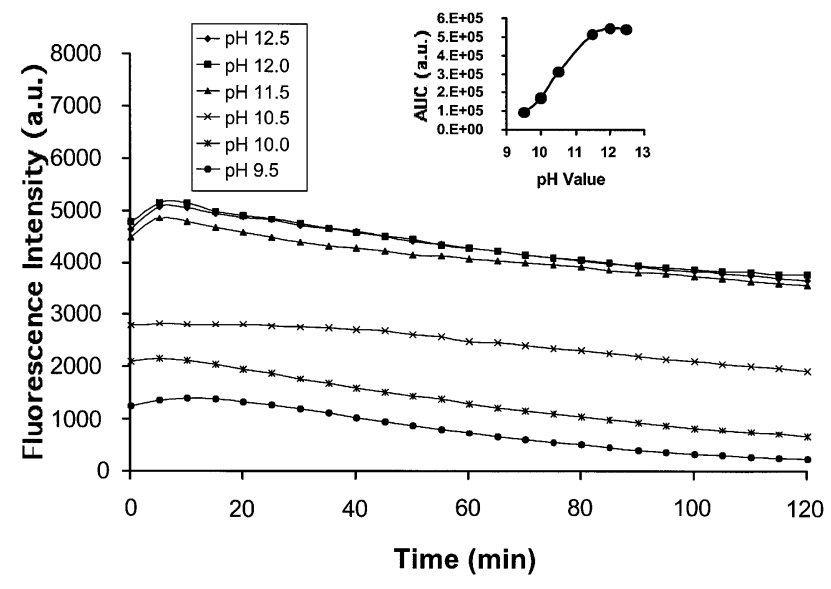

Fig. 2. $\mathrm{pH}$ dependence of NDA-GSH fluorescence over time. GSH $(0.5 \mathrm{mM})$ and NDA $(10 \mathrm{mM})$ were mixed in buffers that had been adjusted to the $\mathrm{pH}$ values indicated and then allowed to incubate for up to $2 \mathrm{~h}$ in a 96 -well microtiter plate. Fluorescence of the conjugate was then assessed during this incubation period. The inset graph shows the calculated area under the fluorescence-time curve for each $\mathrm{pH}$ value tested.

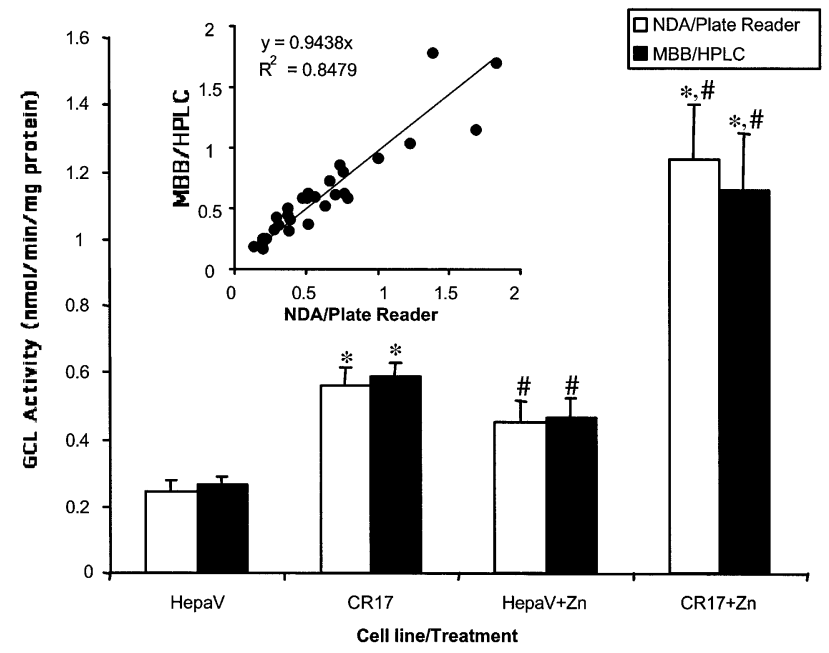

Fig. 3. Comparison of NDA-fluorescence-based and HPLC-based assays of GCL activity using cultured mouse liver cell lines expressing different levels of GCL. Hepa-V cells and CR17 cells were cultured in the absence or presence of $\mathrm{ZnSO}_{4}(50 \mu \mathrm{M})$ for $12 \mathrm{~h}$ prior to harvest. Cells were then analyzed for GCL activity using both NDA-plate reader and MBB-HPLC assays. Inset shows the correlation of these assays for readings taken on individual plates. *Significantly different from similarly treated Hepa-V cells; \#significantly different from untreated cells of the same cell line (Student's $t$ test, $p<0.05$ ).

levels of GCL. CR17 cells have a statistically greater level of GCL activity than Hepa-V cells in both assays, and this activity is further enhanced in both cell lines by zinc pretreatment. There was good agreement in the overall level of GCL activity between the assays, as well as a high degree of correlation between the assays for individual readings (Fig. 3 inset).

Assessment of the GCL activity in mouse liver using NDA-plate reader-based and MBB-HPLC-based assays is shown in Fig. 4. The assays are in very good agree-

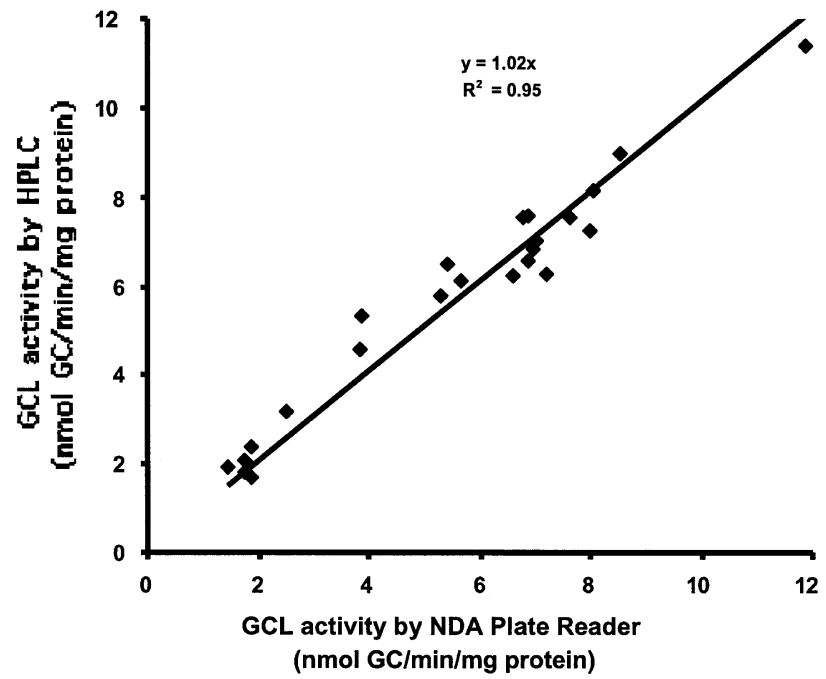

Fig. 4. Comparison of NDA-plate reader-based and MBB-HPLCbased assays of GCL activity using mouse liver homogenates. Liver cytosols from mice treated with varying levels of diethylmaleate were prepared and GCL reactions were carried out as indicated under Materials and methods. After protein precipitation, samples were split and derivatized with NDA or MBB and prepared for either plate reader analysis or HPLC, respectively.

ment as assessed by linear regression analysis $\left(r^{2}=0.95\right.$, slope $=1.02$ ), showing that the NDA-plate reader assay can also be used with tissue samples.

In order to compare the NDA-based assay of GCL activity with the MCB-based assay of GCL activity described by Nelson et al. [21], we simultaneously performed NDA, MCB, and HPLC assays of GCL activity on mouse liver homogenates. As before, we found an excellent correlation between NDA and HPLC assays of GCL activity. However, there was a very weak correlation between MCB and HPLC assays of GCL activity $\left(r^{2}=0.079\right)$.

Finally, we conducted experiments in which we measured GCL activity in normal mouse liver homogenate that had been preincubated with $10 \mathrm{mM}$ buthionine- $S, R$ sulfoximine (BSO), a specific inhibitor of GCL, for $15 \mathrm{~min}$ at $37^{\circ} \mathrm{C}$. GCL activity was completely inhibited by this concentration of BSO, as measured by both NDA and HPLC assays of GCL activity (data not shown). Moreover, BSO at this concentration had no effect on the ability of $\gamma$-GC or GSH to form the NDA conjugate.

\section{Discussion}

We have shown here that NDA can be used to measure both GCL activity and GSH content using a 96-well microtiter plate format. The assay is convenient and sensitive and is in good agreement with GCL activity measurements made using HPLC.

During the course of these experiments, we found that using a $\mathrm{PBS} / \mathrm{SB}$ solution for cultured hepatoma 
cells and at least $45 \mathrm{~min}$ of GCL incubation time yielded reasonably good sensitivity. This allowed for the assessment of GCL activity in approximately 10 to 15 million cells (or 3 to $5 \mathrm{mg} / \mathrm{ml}$ protein for Hepa-1 cells). Dilutions and GCL incubation times necessary for other cell types will depend on the GCL activity and GSH content of those cells, many of which have lower activity than liver cells. It is important to determine the linear range for GCL activity with each cell or tissue type being assayed and to construct a GSH or $\gamma$-GC standard curve with each experiment. However, because the fluorescence intensities of NDA- $\gamma$-GC and NDA-GSH are indistinguishable, we found it most convenient and cost effective to use GSH to construct the standard curve.

We found that homogenization of liver tissue was most conveniently done at a tissue-to-buffer ratio of 1:4 $(\mathrm{w} / \mathrm{v})$, with subsequent $1: 4(\mathrm{v} / \mathrm{v})$ dilution of this homogenate to achieve between 3 and $5 \mathrm{mg} / \mathrm{ml}$ of total protein. Activity measurements on mouse liver tissue required a reaction time of only $10 \mathrm{~min}$ to yield satisfactory sensitivity in the NDA-fluorescence-based assay, as well as a good correlation between this assay and the HPLC assay of GCL activity.

The dilution of both cells and tissue to between 3 and $5 \mathrm{mg} / \mathrm{ml}$ protein is necessary because more concentrated homogenates produce higher concentrations of $\gamma$-GC, and when attempting to derivatize these samples, the additional NDA that is required for complete derivatization results in a polymerization product. We also found that high levels of GSH and $\gamma$-GC (i.e., $>0.5 \mathrm{mM}$ ) in either case resulted in the development of a yellow solution with NDA. This was associated with a quenching of fluorescence, and so it is recommended to not proceed with the assay unless the solution is colorless. Moreover, dilution of the sample lowered the baseline NDA-GSH fluorescence, providing a better signal (NDA-GC)-to-background (NDA-GSH) ratio.

When preparing the Tris/NaOH/NDA derivatization buffer we found that it was necessary to first add the Tris to $\mathrm{NaOH}$, mix thoroughly, and then add the NDA/ $\mathrm{Me}_{2} \mathrm{SO}$ solution, with gentle inversion to mix; otherwise, NDA will polymerize during vortexing. Also, when the derivatization buffer is added to the samples, the plate must not be vortexed, otherwise polymerization of NDA will occur and precipitates will form and this will also interfere with the reading of NDA-GC fluorescence.

We found that the optimal $\mathrm{pH}$ for the reaction between NDA and $\gamma-\mathrm{GC}$ or GSH is 12.5. Cysteine addition results in the formation of a yellow product at lower $\mathrm{pH}$ values and this apparently quenches the NDA- $\gamma$-GC and NDA-GSH fluorescence signals. At $\mathrm{pH} 12.5$ the solution is completely clear with very low cysteine-NDA background fluorescence. Nonetheless, we found that cysteine must be added to all wells (i.e., background GSH wells) because of the slight quenching of fluorescence associated with the cysteine-NDA conjugate.
Addition of $2 \mathrm{mM}$ serine $+20 \mathrm{mM}$ sodium borate to the reaction mixture in order to inhibit any $\gamma$-glutamyltranspeptidase (GGT) present in the samples is an essential step in this protocol. When conducting this assay on cells and tissues containing high GGT activity (e.g., certain tumor cell lines and kidney tissue) it may be advisable to dialyze the sample prior to incubation in the reaction cocktail. Doing so will remove glycine (and GSH) and thus prevent the small amount of conversion of GC to GSH that otherwise occurs through the action of glutathione synthetase. Under these conditions all of the fluorescence measured after derivatization should be due to NDA-GC conjugate formation and thus a direct measure of GCL activity.

Our results with the MCB fluorescence plate reader assay of GCL activity showed a poor correlation with the HPLC assay of GCL activity. Reasons for this could include a considerably high rate of spontaneous formation of cysteine-bimane (which is also fluorescent). This effect can be minimized by lowering the cysteine levels to $0.2 \mathrm{mM}$ in the reaction cocktail (as was done in the assay published by Nelson et al.). However, doing so can result in a limitation in cysteine supply in tissues with high GCL activity, as this concentration of cysteine is near the $K_{\mathrm{m}}$ for the GCL enzyme. The MCB plate assay also requires that the samples be depleted of GSH (in order to lower the baseline fluorescence). This is often done by exposing the cells or animals to GSHdepleting chemicals. Unfortunately, these same chemicals often induce the expression of the GCL enzyme. Thus, the manipulation of the cells or animals in this way can confound the original aim of the experiment, which was to measure GCL activity.

In summary, the NDA-fluorescence-based GCL activity assay is easily conducted, has relatively good sensitivity, and should be applicable to a wide variety of cell and tissue types. Moreover, the ability to measure GSH in the same sample (as background fluorescence in samples not incubated with cysteine) provides an additional benefit when conducting this assay.

\section{Acknowledgments}

This work was supported by NIH Grants 1P42E S04696, 1T32ES07032, P30ES07033, 1P01AG01751, and 1P01CA74184. We thank Dr. R.D. Palmiter, Department of Biochemistry, University of Washington, for kindly providing the metallothionein-promoter plasmid.

\section{References}

[1] A. Meister, Glutathione metabolism, Methods Enzymol. 251 (1995) 3-7. 
[2] C.S. Huang, M.E. Anderson, A. Meister, Amino acid sequence and function of the light subunit of rat kidney gamma-glutamylcysteine synthetase, J. Biol. Chem. 268 (1993) 20578-20583.

[3] C.S. Huang, W. He, A. Meister, M.E. Anderson, Amino acid sequence of rat kidney glutathione synthetase, Proc. Natl. Acad. Sci. USA 92 (1995) 1232-1236.

[4] N. Yan, A. Meister, Amino acid sequence of rat kidney gammaglutamylcysteine synthetase, J. Biol. Chem. 265 (1990) 1588-1593.

[5] J.J. Gipp, C. Chang, R.T. Mulcahy, Cloning and nucleotide sequence of a full-length cDNA for human liver gamma-glutamylcysteine synthetase, Biochem. Biophys. Res. Commun. 185 (1992) 29-35.

[6] J.J. Gipp, H.H. Bailey, R.T. Mulcahy, Cloning and sequencing of the cDNA for the light subunit of human liver gamma-glutamylcysteine synthetase and relative mRNA levels for heavy and light subunits in human normal tissues, Biochem. Biophys. Res. Commun. 206 (1995) 584-589.

[7] L.L. Reid, D. Botta, J. Shao, F.N. Hudson, T.J. Kavanagh, Molecular cloning and sequencing of the cDNA encoding mouse glutamate-cysteine ligase regulatory subunit, Biochim. Biophys. Acta 1353 (1997) 107-110.

[8] L.L. Reid, D. Botta, Y. Lu, E.P. Gallagher, T.J. Kavanagh, Molecular cloning and sequencing of the cDNA encoding the catalytic subunit of mouse glutamate-cysteine ligase, Biochim. Biophys. Acta 1352 (1997) 233-237.

[9] Y. Kang, X. Oiao, O. Jurma, B. Knusel, J.K. Andersen, Cloning/ brain localization of mouse glutamylcysteine synthetase heavy chain mRNA, NeuroReport 8 (1997) 2053-2060.

[10] K. Tsuchiya, R.T. Mulcahy, L.L. Reid, C.M. Disteche, T.J. Kavanagh, Mapping of the glutamate-cysteine ligase catalytic subunit gene (GLCLC) to human chromosome 6p12 and mouse chromosome 9D-E and of the regulatory subunit gene (GLCLR) to human chromosome 1p21-p22 and mouse chromosome 3H1-3, Genomics 30 (1995) 630-632.

[11] A.C. Walsh, W. Li, D.R. Rosen, D.A. Lawrence, Genetic mapping of GLCLC, the human gene encoding the catalytic subunit of gamma-glutamylcysteine synthetase, to chromosome band $6 \mathrm{p} 12$ and characterization of a polymorphic trinucleotide repeat within its $5^{\prime}$ untranslated region, Cytogenet. Cell Genet. 75 (1996) 14-16.

[12] O.W. Griffith, R.T. Mulcahy, The enzymes of glutathione synthesis: gamma-glutamylcysteine synthetase, Adv. Enzymol. Relat. Areas Mol. Biol. 73 (1999) 209-267.

[13] E. Beutler, T. Gelbart, Improved assay of the enzymes of glutathione synthesis: gamma-glutamylcysteine synthetase and glutathione synthetase, Clin. Chim. Acta 158 (1986) 115-123.

[14] C. Birago, E. Marchei, R. Pennino, L. Valvo, Assay of gammaglutamylcysteine synthetase activity in Plasmodium berghei by liquid chromatography with electrochemical detection, J. Pharm. Biomed. Anal. 25 (2001) 759-765.

[15] J.S. Davis, J.B. Balinsky, J.S. Harington, J.B. Shepherd, Assay, purification, properties and mechanism of action of gammaglutamylcysteine synthetase from the liver of the rat and Xenopus laevis, Biochem. J. 133 (1973) 667-678.

[16] G. Nardi, M. Cipollaro, C. Loguercio, Assay of gamma-glutamylcysteine synthetase and glutathione synthetase in erythrocytes by high-performance liquid chromatography with fluorometric detection, J. Chromatogr. 530 (1990) 122-128.

[17] G. Noctor, C.H. Foyer, Simultaneous measurement of foliar glutathione, gamma-glutamylcysteine, and amino acids by highperformance liquid chromatography: comparison with two other assay methods for glutathione, Anal. Biochem. 264 (1998) 98-110.

[18] W.B. Rathbun, H.D. Gilbert, Beta-aminoisobutyrate in the coupled enzymic assay of bovine lens gamma-glutamylcysteine synthetase, Anal. Biochem. 54 (1973) 153-160.

[19] C.C. Yan, R.J. Huxtable, Fluorometric determination of monobromobimane and $o$-phthalaldehyde adducts of gamma-glutamylcysteine and glutathione: application to assay of gammaglutamylcysteinyl synthetase activity and glutathione concentration in liver, J. Chromatogr. B Biomed. Appl. 672 (1995) 217-224.

[20] G.F. Seelig, A. Meister, Glutathione biosynthesis: gamma-glutamylcysteine synthetase from rat kidney, Methods Enzymol. 113 (1985) 379-390.

[21] K.C. Nelson, J.L. Carlson, M.L. Newman, P. Sternberg, D.P. Jones, T.J. Kavanagh, D. Diaz, J. Cai, M. Wu, Effect of dietary inducer dimethylfumarate on glutathione in cultured human retinal pigment epithelial cells, Invest. Ophthalmol. Vis. Sci. 40 (1999) 1927-1935.

[22] O. Orwar, H.A. Fishman, N.E. Ziv, R.H. Scheller, R.N. Zare, Use of 2,3-naphthalenedicarboxaldehyde derivatization for single-cell analysis of glutathione by capillary electrophoresis and histochemical localization by fluorescence microscopy, Anal. Chem. 67 (1995) 4261-4268.

[23] R.D. Palmiter, E.P. Sandgren, D.M. Koeller, R.L. Brinster, Distal regulatory elements from the mouse metallothionein locus stimulate gene expression in transgenic mice, Mol. Cell. Biol. 13 (1993) $5266-5275$

[24] C.C. White, C.M. Krejsa, D.L. Eaton, T.J. Kavanagh, in: M. Maines, L.G. Costa, E. Hodgson, D.J. Reed, I.G. Sipes (Eds.), Current Protocols in Toxicology, Wiley, New York, 1999, Unit 6.5 .

[25] S.A. Thompson, C.C. White, C.M. Krejsa, D. Diaz, J.S. Woods, D.L. Eaton, T.J. Kavanagh, Induction of glutamate-cysteine ligase (gamma-glutamylcysteine synthetase) in the brains of adult female mice subchronically exposed to methylmercury, Toxicol. Lett. 110 (1999) 1-9. 